\title{
Is Brain Aging Retreating to an Infant Brain?
}

\author{
Diego Iacono ${ }^{1,2 *}$ and Gloria C Feltis ${ }^{1}$ \\ ${ }^{1}$ Neuropathology Research, Biomedical Research Institute of New Jersey, Cedar Knolls, NJ, USA \\ ${ }^{2}$ MidAtlantic Neonatology Associates, Morristown, NJ, USA
}

*Corresponding author: Diego Iacono, Biomedical Research Institute of New Jersey, BRInj, and MANA, Atlantic Health System, NJ, 140 East Hanover Avenue, Cedar Knolls, NJ 07927, USA

To Cite This Article: Iacono D, Feltis GC. Is Brain Aging Retreating to an Infant Brain?. Am J Biomed Sci \& Res. 2019 - 1(1). AJBSR.MS.ID.000505. DOI: 10.34297/AJBSR.2019.01.000505

Received 㣋: January 06, 2019; Published 眥: January 08, 2019

\section{Opinion}

Brain aging considered in its normality that is, in the absence of obvious incapacities to independently perform common daily activities across the different human domains (sensorimotor, cognition, emotional, behavioural tasks), can be defined as a timerelated biological and psychological process that mainly involve the speed employed to achieve a specific task or total amount of operations that a single subject can simultaneously perform in a given period of time in comparison to younger ages. In this context then, normal brain aging capacities do not look like so dissimilar from the characteristic features of the brain capacities during infancy. Both periods of life, elderly (>75 years) and infancy (024 months after full-term birth), are normally characterized by neurobiological changes that have, respectively, a forward and a backward impact on the rest of the lifespan.

While there are different possible overlapping characteristic in terms of global performance between elder and infants brains, still, it is not known if normal brain aging and normal brain development are indeed a going back and forth process in terms of neurobiological changes [1], genetic programming [2], and if the one and the other process are indeed modulated by environmental factors such as nutrition [3], familial and social environment, emotional stress (either negative or positive stress), ego-syntonic activities (school, job, leisure activities, affection, etc.). Newer findings are increasingly showing that the vulnerability of the brain during aging, as measured in a specific individual, could indeed represent the global or region-specific brain functional or dysfunctional outcome resulting from the accumulating interactions between both endogenous and exogenous components of the entire life that have mutually interrelated during the previous decades. While during elderly these interactions are a load that "push down" certain functions (e.g. memory storage capacities), during infancy the identical factors could "push up" the central nervous system to increase the level of complexity of the wiring until the individual-based genetic and environmental potential has been reached. Here, we have considered only the normal brain aging and developmental domains. However, the same type of considerations should be probably taken in account for agingrelated neurodegenerative processes, as well as for altered neurodevelopmental processes [4,5].

Which are the initial (early-life) imprinting genetic and nongenetic factors that could determine age-onset, natural speed progression and severity of an aging-related brain disease (e.g. Alzheimer's disease) in the absence of a genetic mutation? There are not clear or definitive answers, yet, to this basic question.

A topic of paramount importance in future neurogeriatrics and neurodevelopmental investigations will be to define the neurobiological foundations for the possible similarities and differences between these two periods of the human life. It is our opinion though that, not only the progression in the neurodevelopmental field could largely inform the neuro-geriatric field but that even the neuro-geriatric field could generate knowledge that might help to better understand the early events of the brain life. If coinciding, the molecular underpinnings of the normal brain aging and brain development will potentially generate a wide spectrum of pathways on which possibly interact through future interventions either pharmacological as nonpharmacological for the maintenance of the brain function during normal aging and extreme aging ( $>100$ years) or for the potentiation of the brain functions and capacities [6] in the future generations.

\section{References}

1. Walhovd KB, Fjell AM, Espeseth T (2014) Cognitive decline and brain pathology in aging--need for a dimensional, lifespan and systems vulnerability view. Scand J Psychol 55(3): 244-254.

2. Fjell AM, Grydeland H, Krogsrud SK, Amlien I, Rohani DA, et al. (2015) Development and aging of cortical thickness correspond to genetic organization patterns. Proc Natl Acad Sci USA 112(50): 15462-15467. 
3. Prinelli F, Fratiglioni L, Musicco M, Johansson I, Adorni F (2018) The impact of nutrient-based dietary patterns on cognitive decline in older adults. Clin Nutr S0261-5614(18): 32580-32589.

4. Douaud G, Groves AR, Tamnes CK, Westlye LT, Duff EP, et al. (2014) A common brain network links development, aging, and vulnerability to disease. Proc Natl Acad Sci USA 111(49): 17648-17653.
5. Kovacs GG, Adle-Biassette H, Milenkovic I, Cipriani S, van Scheppingen J, et al. (2014) Linking pathways in the developing and aging brain with neurodegeneration. Neuroscience 269: 152-172.

6. Gomes-Osman J, Indahlastari A, Fried PJ, Cabral DLF, Rice J, et al. (2018) Non-invasive Brain Stimulation: Probing Intracortical Circuits and Improving Cognition in the Aging Brain. Front Aging Neurosci 10: 177. 